\title{
PESQUISA FITOQUÍMICA EM PANCS DA REGIÃO DOS CAMPOS GERAIS
}

\section{PHYTOCHEMICAL RESEARCH IN PANCS OF GENERAL FIELDS REGION}

\author{
Anna Caroline Lopes', Amanda de Moraes', Dra. Vanessa Barbosa Bobek²
}

1 - CESCAGE - Curso superior de Farmácia

2 - CESCAGE - Docente do curso de Farmácia

Autor para correspondência: amandinha.moraes53@gmail.com

\begin{abstract}
RESUMO: O termo PANC engloba plantas ou partes delas que não participam da cadeia produtiva, e que mesmo encontradas abundantemente não tem suas propriedades exploradas pela população. As PANC'S trazem em sua composição nutrientes necessários para o organismo, e algumas ainda apresentam propriedades medicinais e compostos bioativos como os antioxidantes, que ajudam na prevenção e tratamento de doenças. Este trabalho teve como objetivo avaliar a composição fitoquímica, do Picão-Preto (Bidens pilosa) e da folha de batata doce (Ipomoea batatas), PANCs conhecidas popularmente na região Sul do Brasil, avaliando a ocorrência de taninos, flavonoides, saponinas e alcaloides através de testes de caracterização vegetal, utilizando-se como parâmetro os testes estabelecidos pela Sociedade Brasileira de Farmacognosia (SBF). Todas as amostras analisadas apresentaram resultado positivo para flavonoides e taninos, e resultado negativo para saponinas. No caso dos alcaloides, as batatas BD1 e BD3 e o picão preto apresentaram resultado positivo e as demais resultado negativo.
\end{abstract}

Palavras-chave: Bidens, Ipomoea, pesquisa fitoquímica

ABSTRACT: The term PANC encompasses plants or parts of them that do not participate in the production chain, and which even found abundantly do not have their properties explored by the population. The PANC'S bring in its composition nutrients needed for the body, and some even have medicinal properties and bioactive compounds such as antioxidants, which help in the prevention and treatment of diseases. . The objective of this work was to evaluate the phytochemical composition of Picão-preto (Bidens pilosa) and sweet potato leaves (Ipomoea potatoes), PANCs popularly known in southern Brazil, evaluating the occurrence of tannins, flavonoids, saponins and alkaloids through plant characterization tests, using as parameters the tests established by the Brazilian Society of Pharmacognosy (BSP). All samples analyzed were positive for flavonoids and tannins, and negative for saponins. In the case of alkaloids, potatoes BD1 and BD3 and picao preto presented positive results and the other negative results.

Keywords: Bidens, Ipomoea, phytochemical research

\section{INTRODUÇÃO}

O Brasil é o país com a maior biodiversidade do planeta, o que confere a ele uma imensa variedade de biomas, e uma fauna e flora de riquezas incontáveis (BRASIL, 2019).

No entanto, apesar da abundância alimentícia e medicinal encontrada no país, pouco do que se tem é estudado, o que faz com que a atividade farmacológica de uma parcela dessas plantas não seja conhecida, como é o caso das PANC's (Plantas Alimentícias Não Convencionais) (KINUPP, 2009). As PANC's são plantas ou partes delas, sejam alimentícias ou medicinais, que poderiam ser consumidas, mas não são utilizadas com frequência pela população (OLIVEIRA et al., 2018).

Contudo, para se classificar uma planta como alimentícia não convencional faz-se necessário uma análise do seu hábito de consumo. Determinado vegetal comumente 
utilizado em uma área, pode ser denominado como PANC em outra região (FONSECA et al., 2018).

Na região Sul do Brasil, algumas plantas ganham destaque por sua boa adaptação em todas as estações climáticas, podendo ser cultivadas ou de crescimento espontâneo, como é o caso da batata-doce (Ipomoea batatas L. lam.) e do Picão (Bidens pilosa) (INSTITUTO KAIRÓS, 2017).

Presente em praticamente todo o território brasileiro mas pouco utilizada no Sul, o Picão-Preto (Bidens pilosa) é um exemplo de PANC com propriedades farmacêuticas. É uma planta herbácea, de desenvolvimento rápido e que pode ser encontrada durante todas as estações do ano. Suas folhas têm coloração verde e podem ou não ter pelos (SANTOS; CURY, 2011).

É uma planta daninha bastante agressiva e possui propriedades fitotóxicas a outros vegetais e microrganismos. Tem um reconhecido valor medicinal e quase todas as partes da planta são utilizadas nas preparações, mas atualmente, a Farmacopeia não traz nenhuma monografia oficial para a Bidens pilosa.

A folha da batata doce (Ipomoea batatas L. lam), também é um exemplo de PANC com propriedades farmacêuticas pouco exploradas pela população. É uma planta trepadeira de fácil adaptação em diversas regiões climáticas, muito encontrada em culturas agrícolas. No entanto, pode crescer em regiões não cultivadas, como lotes vagos e florestas. Suas folhas são lisas e tem formato de coração (JESUS et al., 2018).

A batata doce tem um ciclo curto de reprodução e alto valor nutricional, é um alimento rico em carboidratos, fibras e vitaminas. Suas folhas já são utilizadas na medicina popular e testes realizados por pesquisadores já encontraram atividades farmacológicas, porém esses ainda não estão descritos na Farmacopéia Brasileira.

É conhecido como metabólito secundário o grupo de compostos orgânicos que não estão diretamente ligados à sobrevivência do vegetal, mas que apresenta propriedades importantes para a adaptação desse ao meio (PEREIRA; CARDOSO, 2012).

Uma das formas de avaliar e identificar esses compostos que formam o grupo de metabólitos secundários relevante das plantas é a Pesquisa Fitoquímica (SILVA et al., 2010). Ela é importante para identificar metabólitos nas espécies vegetais e para avaliar a presença dos mesmos. Com a pesquisa é possível identificar diversos compostos químicos, inclusive os responsáveis pela atividade farmacológica das PANCs (BESSA et al., 2013). 


\section{MATERIAL E MÉTODOS}

A coleta de B. pilosa foi realizada em 07/08/2019 na região dos campos gerais (coordernadas WV6V+3F Uvaranas, Ponta Grossa - PR). A exsicata foi identificada e depositada no Herbário da Universidade Estadual de Ponta Grossa (UEPG) e aguarda número de registro.

As amostras de I. batatas de 5 variedades diferentes foram gentilmente cedidas pelo Prof. Dr. Jaime Alberti Gomes do departamento de Agronomia daUniversidade Estadual de Ponta Grossa (UEPG).

Para a caracterização da droga vegetal foi realizada a análise fitoquímica, avaliando a ocorrência de taninos, flavonoides, saponinas e alcaloides, utilizando-se como parâmetro os testes estabelecidos pela Sociedade Brasileira de Farmacognosia (SBF).

\subsection{Pesquisa fitoquímica}

\subsubsection{TANINOS}

Em um béquer, foram pesados $3 \mathrm{~g}$ da planta pulverizada. Foram adicionados $50 \mathrm{~mL}$ de água destilada e fervido por 2 minutos. A mistura foi filtrada em papel filtro, e o filtrado foi utilizado para as reações de caracterização.

\section{- Reação com cloreto férrico}

Foi colocado $1 \mathrm{~mL}$ da solução extrativa em tubo de ensaio e adicionado 3 gotas de cloreto férrico $2 \%$ (escorrendo pela parede do tubo).

\section{- Reação com acetato de chumbo}

Foi colocado $3 \mathrm{~mL}$ da solução extrativa no tubo de ensaio e adicionado 3 gotas da solução de acetato de chumbo $10 \%$.

\section{- Reação com acetato de chumbo e ácido acético glacial}

Foi colocado $3 \mathrm{~mL}$ da solução extrativa no tubo de ensaio e adicionado $2 \mathrm{~mL}$ de 
ácido acético glacial $10 \%$ e após, $3 \mathrm{~mL}$ de acetato de chumbo $10 \%$.

\subsubsection{FLAVONOIDES}

No béquer, foram pesados $3 \mathrm{~g}$ da planta pulverizada e adicionado $15 \mathrm{~mL}$ de etanol $70 \%$. A mistura foi fervida, com cuidado, por 2 minutos e filtrada em papel filtro. $O$ filtrado foi utilizado para as reações.

\section{- Reação de Shinoda}

Foram colocados fragmentos de magnésio metálico no tubo de ensaio. Adicionaouse $5 \mathrm{~mL}$ da solução extrativa. Foi acrescentado, lentamente, pela parede do tubo, 1,0 mL de ácido clorídrico concentrado.

\section{- Reação com cloreto férrico}

$1 \mathrm{~mL}$ da solução extrativa foi diluído em $4 \mathrm{~mL}$ de água destilada. A solução obtida foi colocada no tubo de ensaio e foi adicionado 1 gota de cloreto férrico $2 \%$ (escorrendo pela parede do tubo).

\section{- Reação com hidróxido de sódio}

$1 \mathrm{~mL}$ da solução extrativa foi diluído em $4 \mathrm{~mL}$ de água destilada. A solução obtida foi colocada no tubo de ensaio e foram adicionadas 2 gotas de hidróxido de sódio $5 \%$.

\subsubsection{SAPONINAS}

Ferveu-se $2 \mathrm{~g}$ da droga com $10 \mathrm{~mL}$ de água destilada. A solução extrativa foi transferida para um tubo de ensaio, tampada e agitada vigorosamente por 20 segundos. A altura da espuma foi marcada com caneta. Deixou-se em repouso e após 10 minutos foi feita a observação.

\subsubsection{ALCALOIDES}

Aqueceu-se $4 \mathrm{~g}$ da planta pulverizada e $20 \mathrm{~mL}$ de ácido clorídrico a $10 \%$, até início 
da fervura. Deixou-se esfriar e a solução foi filtrada em um funil de separação. $O$ filtrado foi alcalinizado com solução de hidróxido de amônio, utilizando papel tornassol como indicador.

Extraiu-se a solução alcalina com $10 \mathrm{~mL}$ de clorofórmio. A fase orgânica foi separada. Filtrou-se em algodão hidrófilo com sulfato de sódio anidro. O clorofórmio foi evaporado em banho Maria. Redissolveu-se o resíduo resultante em $8 \mathrm{~mL}$ de ácido clorídrico a $10 \%$.

\section{- Reativos para alcaloides}

O conteúdo foi dividido em 3 tubos de ensaio e o teste foi realizado com 2 gotas dos reativos para alcaloides (Dragendorff).

\section{RESULTADOS E DISCUSSÃO}

\subsection{Pesquisa fitoquímica}

A pesquisa fitoquímica preliminar permite fazer a detecção dos principais grupos de metabólitos secundários presentes na espécie em estudo, buscando também os marcadores químicos da espécie, dados que auxiliam no controle de qualidade, também servindo de base para a purificação, isolamento, caracterização e síntese de compostos. Os resultados obtidos estão descritos na TABELA 1.

TABELA 1 - RESULTADO DA PESQUISA FITOQUÍMICA

\begin{tabular}{|c|c|c|c|c|c|c|}
\hline Classe de metabólito & B1 & B2 & B3 & B4 & B5 & B. pilosa \\
\hline Alcaloides & + & - & + & - & - & + \\
\hline Flavonoides & + & + & + & + & + & + \\
\hline Taninos hidrolisáveis & + & + & + & + & + & + \\
\hline Taninos condensados & + & + & + & + & + & + \\
\hline Saponinas & - & - & - & - & - & - \\
\hline
\end{tabular}

NOTA: (+) presença, (-) ausência.

Os alcaloides são encontrados nos vegetais em forma de substâncias 
nitrogenadas, sendo na forma combinada com ácidos orgânicos ou então em forma livre, que é insolúvel em meio aquoso e solúvel em solventes orgânicos (SOCIEDADE BRASILEIRA DE FARMACOGNOSIA, 2009).

Para análise da presença de alcaloides foi realizado testes com reativos gerais de alcaloides (RGA), sendo que eles formam turvação a precipitação em meio ácido. Apenas as variedades de Ipomoea 1 e 3 apresentaram resultados positivos para a presença de alcaloides. Entre os testes para alcaloides utilizados no experimento, o que apresentou reação positiva foi o teste com o reagente de Dragendorff. Os alcaloides têm sido efetivos em seus efeitos medicinais e são encontrados em diversas espécies de plantas, sendo que exercem papel importante como substâncias de defesa (SIMÕES et al., 2017). B. pilosa apresentou seu resultado positivo para a presença de alcaloides em concordância com o descrito na literatura (BRASILIA, 2015).

Os flavonoides são compostos naturais, derivados da benzo-y-pirona e constituem o maior grupo de compostos fenólicos naturais, sendo por isso muito utilizados como marcadores de quimiossistemáticos. São conhecidos hoje, mais 2000 flavonoides, que podem ocorrer no seu estado livre ou como O-glicosídeos que é o mais comum. Na terapêutica, sua presença está relacionada com atividade anti-inflamatória, antialérgica e vasoprotetora (SOCIEDADE BRASILEIRA DE FARMACOGNOSIA, 2019).

No trabalho realizado por Soares (2013), a pesquisa fitoquímica clássica, realizada para a detecção dos constituintes químicos dos extratos brutos hidroalcoólicos das folhas de I. batatas revelou positividade para a presença de compostos fenólicos gerais, flavonas, flavonol, xantonas e catequinas. Estes resultados corroboram dados gerados por estudos anteriores com folhas desta espécie. Pochapski et al. (2011) relataram a detecção destes metabolitos em diferentes meios extrativos com concentrações variadas. Outros trabalhos relatam também que tais compostos se constituem os principais componentes biologicamente ativos da planta (Li et al., 2000).

$\mathrm{Na}$ pesquisa realizada por Leite (2009), percebeu-se que os extratos das variedades de $I$. batatas escolhidas aleatoriamente para a corrida cromatográfica, havia diferença em sua constituição flavonoidica. Isto é explicado pelo fato de os compostos fenólicos poderem variar até mesmo dentro de espécies e as variedades utilizadas na corrida cromatográfica apresentarem algumas peculiaridades fisiológicas.

A espécie $B$. pilosa é uma fonte de compostos fitoquímicos, especialmente flavonoides e poliacetilenos (BARTOLOME; VILLASENOR; YANG, 2013). Em um artigo de revisão, foram descritos 198 compostos para a espécie (SILVA et al., 2011). Foram descritos 
ainda, em outra revisão, diversos compostos isolados da espécie, sendo estas substâncias das classes dos produtos naturais alifáticos, flavonoides, terpenoides, fenilpropanoides e compostos aromáticos (BARTOLOME; VILLASENOR; YANG, 2013).

Os taninos são compostos fenólicos presentes em inúmeros vegetais, capazes de formar complexos insolúveis com proteínas, alcaloides e gelatinas. A maioria dos frutos e derivados vegetais com essa classe de metabólito secundário tem como característica a adstringência, uma vez que precipita glucoproteínas salivares, ocasionando a perda de lubrificação. Na terapêutica, sua presença tem sido associada com atividade antiinflamatória, cicatrizante, e até inibição da transcriptase reversa em HIV (MONTEIRO, et al 2005).

Os taninos podem ser classificados em dois grupos, sendo eles os hidrolisáveis e condensados. Os hidrolisáveis têm em sua formação um resíduo de glicose central que é ligada à muitas moléculas de ácido fenólicos, suas ligações podem ser por ester hidrolisáveis por ácidos ou enzimas. Os condensados são moléculas mais resistentes, é composto por uma estrutura polimérica do flavan-3ol, como a catequina ou do flavan-3,4diol (SOCIEDADE BRASILEIRA DE FARMACOGNOSIA, 2009).

Em todas as amostras analisadas, foi detectada a presença de taninos hidrolisáveis e condensados. Santos (2015) realizou o doseamento de taninos nos extratos brutos e frações acetato de etila e butanólica obtidas através de diferentes cultivos de I. batatas e encontrou os maiores teores nos extratos brutos e na fração acetato de etila, sendo 136,75 $\mathrm{mg} / \mathrm{g}$ e $139,25 \mathrm{mg} / \mathrm{g}$ respectivamente.

A espécie B.pilosa apresenta diferença na presença dos seus composto secundários sendo de acordo com a parte da planta analisada. Por exemplo, o extratodas sementes apresentou saponinas, alcaloides e taninos, e o extrato de caule teve presença de saponina e tanino. Já o extrato de folhas foi dectado presença de taninos, saponinas, alcaloides e flavonoides (CHAVASCO et al., 2014).

As saponinas são compostos nitrogenados, que quando em contato com a água se dissolvem e diminuem a tensão superficial do líquido, originando soluções afrógenas (espumantes). Apresenta propriedades de produzir hemólise e emulsionar óleos. Podem ser classificadas quimicamente em glicosídeos saponosídicos do tipo esteroide e triterpênico (SOCIEDADE BRASILEIRA DE FARMACOGNOSIA, 2019).

Para a pesquisa de saponinas o método utilizado foi o teste qualitativo da espuma, onde todas as amostras de I. batatas tiveram resultado negativo, Entretanto Pochapski (2010) observou a formação de espuma persistente nas frações acetato de etila e na fração 
alcoólica do extrato bruto hidroalcólico de I. batatas que caracterizou a presença de glicosídeos saponínicos.

A espécie B. pilosa também apresentou resultado negativo para saponinas, entretanto no estudo realizado por Chavasco et al. (2014) foi detectada a presença desse metabólito nos extratos de caules e sementes. Segundo Gobbo- Neto; Lopes (2007), as condições ambientais alteram a produção e concentração das substâncias ou metabólitos secundários nas plantas, como composição atmosférica, fase de desenvolvimento das plantas, altitude, nutrientes do solo, temperatura, radiação ultravioleta, disponibilidade de água e sazonalidade. Sendo assim uma mesma espécie que é cultivada de maneira diferente, pode ter diferença no acumulo de substancias e diferença na síntese.

\section{CONCLUSÃO}

Os testes realizados permitiram demonstrar a presença de metabólitos secundários como flavonoides, alcaloides, taninos e saponinas nas espécies analisadas, o que é de grande importância, visto que esses compostos estão diretamente ligados aos efeitos farmacológicos que as plantas podem exercer.

\section{REFERÊNCIAS}

BESSA, N.G.F.de. et al. Prospecção fitoquímica preliminar de plantas nativas do cerrado de uso popular medicinal pela comunidade rural do assentamento vale verde - Tocantins. Revista Brasileira Plantas Medicinais, Campinas, v.15, n.4, supl.I, p.692-707, 2013.

BRASIL. Ministério do Meio Ambiente. Biodiversidade Brasileira 2019. Disponível em http://www.mma.gov.br/biodiversidade/biodiversidade-brasileira Acesso em: 15 mar. 2019.

Chavasco JM, Prado E Feliphe BH, Cerdeira CD, Leandro FD, Coelho LF, da Silva JJ, et al. Evaluation of antimicrobial and cytotoxic activities of plant extracts from southern Minas Gerais cerrado. Rev Inst Med Trop Sao Paulo. 2014;56(1):13-20.

FONSECA, C. et.al. A importância das Plantas Alimentícias Não Convencionais (PANCS) para a sustentabilidade dos sistemas de produção de base ecológica. In: I Congresso Latino-Americano, X Congresso Brasileiro, V Seminário do DF e entorno, set. 2017, Brasília. 
Cadernos de Agroecologia, Brasília, ISSN 2236-7934 - Anais do VI CLAA, X CBA e V SEMDF - Vol. 13, N 1, Jul. 2018.

GOBBO-NETO, et al. Plantas medicinais: fatores de influência no conteúdo de metabólitos secundários. Quim. Nova, Vol. 30, No. 2, 374-381, 2007

INSTITUTO KAIRÓS. Guia Prático sobre PANCs: plantas alimentícias não convencionais. Câmara Brasileira do Livro, 1 ed. São Paulo, 2017 p.16-34

ISLAM, S. Medicinal and Nutritional Qualities of Sweetpotato Tops and Leaves. Plant Science, v. 4, p. 3- 7, 2009.

JESUS. M. et. al. Teor de carotenoides de três variedades de batata-doce (Ipomoea batatas L.) cultivadas sob sistemas de produção orgânico e convencional. Cadernos 23 de Agroecologia - ISSN 2236-7934 - Anais do VI CLAA, X CBA e V SEMDF Vol. 13, № 1, Jul. 2018.

MONTEIRO, et al. Taninos: uma abordagem da química à ecologia. Quim. Nova, Vol. 28, No. 5, 892-896, 2005.

OLIVEIRA et.al. Plantas Alimentícias Não Convencionais (PANCs) no Município de Campo Grande/MS: Conhecimento Popular, Consumo e Comércio. Cadernos de Agroecologia, Campo Grande, ISSN 2236 -7934 - V. 13, N. 2, Dez. 2018.

PEREIRA, R.J.; CARDOSO, M, G. Metabólitos secundários vegetais e benefícios antioxidantes, Palmas, Vol. 3, N. 4: pp. 146-152, Novembro 2012. Disponível em: <https://www.todafruta.com.br/wp-content/uploads/2016/09/Metab\%C3\%B3litossecund\%C3\%A1rios-ARTIGO.pdf> Acesso em 17/09/2019

POCHAPSKI, Márcia Thaís. Estudo dos efeitos da Ipomoea batatas (L.) Lam. na resposta inflamatória e no biofilme dental. 2010. 84 f. Tese (doutorado) - Universidade Estadual de Campinas, Faculdade de Odontologia de Piracicaba, Piracicaba, SP. Disponível em: <http://www.repositorio.unicamp.br/handle/REPOSIP/288949>. Acesso em: 14 de novembro de 2019 
SANTOS, J.B. e CURY, J.P., Picão-preto: uma planta daninha especial em solos tropicais. Planta Daninha, Viçosa-MG, v. 29, p. 1159-1171, 2011. Número Especial Silva FL, Fischer DC, Tavares JF, Silva MS, de Athayde-Filho PF, Barbosa-Filho JM. Compilation of secondary metabolites from Bidens pilosa L. Molecules. 2011;16(2):1070- 102.

SILVA, N. L. A. et.al. Triagem Fitoquímica de Plantas de Cerrado, da Área de Proteção Ambiental Municipal do Inhamum, Caxias, Maranhão. Scientia plena, V. 6, N. 2, Jan. 2010

SIMÕES, et.al. Farmacognosia: do produto natural ao medicamento [recurso eletrônico]. Porto Alegre: Artmed, 2017. Disponível em: https://books.google.com.br/books ?hl=pt-BR\&lr=\&id=uo5vDQAAQBAJ\&oi=fnd \&pg=PT4\&dq=SIM\%C3\%95ES, +C.+M.+O.\% 3B+SCHENKEL,+E.+P.\%3B+MELLO,+J.+C.+P.\%3B+MENTZ,+L.+A.\%3B+PETROVICK, + P.+R.\&ots=1 CbHtZnomd\&sig=fbOyZEtQ-dxVdGbQlf2KQNMNXc0\#v=Onepage\&q\&f=false. Acesso em 13 de novembro de 2019.

SOARES, I. M. Determinação do conteúdo fenólico, flavonoides e atividade antioxidante nas folhas de dez cultivares de Ipomoea batatas (L.) Lam. desenvolvidas para produção industrial de etanol. 124 f. Dissertação (Mestrado) - Universidade Federal do Tocantins, Programa de Pós-Graduação em Agroenergia, 2013.

SOCIEDADE BRASILEIRA DE FARMACOGNOSIA, 2009 Flavonoides e antocianos. Disponível em: <http://www.sbfgnosia.org.br/Ensino/flavonoides_e_antocianinos.html> Acesso em: 14/06/19

SOCIEDADE BRASILEIRA DE FARMACOGNOSIA, 2009 Pesquisa de alcaloides. Disponível em: < http://www.sbfgnosia.org.br/Ensino/alcaloides.html> Acesso em: 14/06/19

SOCIEDADE BRASILEIRA DE FARMACOGNOSIA, Pesquisa de saponinas - teste qualitativo da espuma. Disponível em: <http://www.sbfgnosia.org.br/Ensino/Saponinas.html> Acesso em: 14/06/19

SOCIEDADE BRASILEIRA DE FARMACOGNOSIA, Taninos. Disponível em: <http://www.sbfgnosia.org.br/Ensino/taninos.html> Acesso em: 14/06/19 